\title{
Toward High-Throughput Fungal Electroculturomics and New Omics Methodologies in 21st-Century Microbiology and Ecology
}

\author{
Andreas Stathoulias, ${ }^{1}$ Aphroditi Milioni, ${ }^{2}$ Stavroula Kritikou, ${ }^{2}$ Aikaterini Karmakolia, ${ }^{3}$ \\ Stavroula Goudoudaki, ${ }^{3}$ Stavroula Siamoglou, ${ }^{1}$ Constantinos Chassomeris, ${ }^{1}$ Stamatis Vassilakis, \\ Kariofyllis Karamperis, Aristea Velegraki, ${ }^{1}$ Cleo Anastassopoulou, ${ }^{2}$ Yiannis Manoussopoulos, ${ }^{3}$ \\ George P. Patrinos, and Manousos E. Kambouris ${ }^{1}$
}

\begin{abstract}
Modern microbiology and drug development are in a watershed moment with the advent of electroceuticals. In addition to genomics, electrical impulses in an organism are believed to contribute to tissue and cellular plasticity. Hence, electroceuticals or bioelectronics offers the promise to identify innovative approaches to treat human diseases. However, applications toward electromicrobiology are still limited and rare, despite the high potential to innovate the fields of both microbiology and therapeutics. For example, electric modalities for manipulating microbial growth are highly sustainable; can be combined with biopharmaceuticals, probiotics, and pharmacobiotics; and, thus, are well poised for use in medicine, public health, and ecology and diverse industries. We report here the introduction of a new research framework and technology platform for electroculturomics, by coupling standard solid-state mycological cultures with conductive treatment using a conformité Européene (CE-)-certified medical ionophoresis device. We share our experience with a diverse range of fungi that have been treated with the electroculturomics approach reported herein. We suggest that this line of inquiry can be extended to electrotranscriptomics and electrometabolomics by deploying electroculturomics in tandem with multi-omics approaches in the future. This article makes a specific contribution to fungal microbiology, and a broader contribution to advance the theory and practice of the field of electroculturomics emerging in 21 st-century microbiology and ecology research.
\end{abstract}

Keywords: conductive electrostimulation, culturomics, fungal electroculturomics, electroceuticals, microbiology, bioelectronics

\section{Introduction}

$\mathbf{I}_{\mathrm{r}}^{\mathrm{T}}$ T IS ANTICIPATED THAT THE IMPORTANCE OF MICROBIOTA RESEARCH will markedly increase in all sectors of life sciences over the next decade (Frinking et al., 2016; Kambouris et al., 2018a, 2018b, 2018c; Koblentz, 2017). With this realization, the concept of the microbiome has become more established and accepted many years after its inception (Whipps et al., 1988).

From the microbial apocalypse (Wiles, 2015) to the microbial or microbiome revolution (Blaser, 2014; Pikaar et al., 2017), the manipulation of the growth of microbiota is one of the hottest issues, especially as the conventional approaches, the cocktails of chemical and biochemical compounds, become increasingly unaffordable and definitively unsustainable (Kambouris et al., 2017, 2018c).

In this spirit, the microbiota of medicinal, economic, and environmental interest have been sought after in the course of a century, and studied by a number of electromagnetic modalities to determine their putative stimulatory or inhibitory effects, transitional dynamics, and so as to establish the dose-response relationships regarding their primary and secondary metabolism. The interaction of electrical modalities with microbiota was recently observed during sessions of

\footnotetext{
${ }^{1}$ Laboratory of Pharmacogenomics and Individualized Therapy, Department of Pharmacy, School of Health Sciences, University of Patras, Patras, Greece.

${ }^{2}$ Department of Microbiology, School of Medicine, National and Kapodistrian University of Athens, Athens, Greece.

${ }^{3}$ Laboratory of Virology, Plant Protection Division of Patras, ELGO-Demeter, Patras, Greece.
} 
wound healing by electrostimulation (Poltawski and Watson, 2009). Such treatment has been shown to speed up healing (Kambouris et al., 2014), partly through decontaminating the wound site (Kloth, 2005; Ramadhinara and Poulas, 2013; Rowley et al., 1974).

A new era of therapeutics innovation was officially inaugurated with the introduction of "electroceuticals" (Famm et al., 2013) or bioelectronics as concepts intended as an analogue to "pharmaceuticals" (Kambouris et al., $2014,2017)$ so as to describe the diverse modalities differing in principle, form, cumulative dose, required treatment duration, and other parameters. Their common denominator is the elicitation of biological effects in an organism, by means of external electrical stimuli, for restoring impaired biological functions.

Of course, this new era has already been in the works and has been advancing with many diverse, intermittent, inconsistent, and, at times, stray efforts since the mid-1990s (Kloth, 2005; Poltawski and Watson, 2009), which, however, achieved a critical mass of scholarship so as to achieve recognition, including by health care insurance providers as a valid approach (Healthcare, 2008).

Notably, the electromagnetic stimulus may be a field, which may result in the initiation of a current ("inductive coupling") or in polarization. Alternatively, it may be the routing of an actual current through the target moiety, forming part of an electric circuit ("conductive coupling").

\section{Overarching theory and background for the present study}

Because electroceuticals, bioelectronics, and the field of electroculturomics are relatively new scientific frontiers, we present here an overarching background for this study.

In this study, a conductive format, implemented by a conformité Européene (CE-)-certified instrument of medical electrotherapy (Class IIa equipment according to Directive 93/42/EEC, annexed IX rule 9), intended mainly for orthopedics use, has been tested on a series of fungi in solid culture and ambient conditions. The overarching aim was to introduce the field of electroculturomics (Kambouris, 2020) and establish the baseline experimental conditions to inform future fundamental electroculturomics research and development. In this regard, a first objective was to determine the proper form of controls in order to establish a comparison basis for the treated samples.

The second objective was to identify variables affecting dose-response curves. Such knowledge will be governing the methodology of experimental procedures of later, fundamental research in the near future. Although the multi-omics nature of the electroculturomics concept implies a high degree of interdependence among the levels of study (i.e., genomics and post-genomics), a relatively limited number of variables was selected to study in rather simple formats as a baseline to inform future higher dimensional work.

Our focus points were the intensity of the current and the frequency of the treatment. They may be considered as two variables that define the accumulative electric charge used. Another such point has been the fungal species or rather taxa since the variability of fungal responses to both antifungal agents and growth factors/substrates is well known. In the course of this work, we checked for additional factors that are pertinent to electroculturomics, such as culture substrate and the angle/polarity of the electro-treatment.

The last study objective was to proceed to an indicative case of fundamental research in area(s) identified by the initial experimental work presented here, to establish a basis of electroculturomics data so as to commence relevant applications. We note that the causal/mechanistic explanation of the observed results is a task for subsequent research in our electroculturomics research program as this nascent field continues to advance in the near future.

To increase the probability of observing a broad array of biological effects for further future study, an inclusive approach was selected, with a variety of thallic forms, species, phyla, and on different substrates; an agnostic and multiparametric approach that is typical for culturomics (Kambouris and Velegraki, 2020). For the initial development of the approach, flexibility of application is considered as being more important than the integration of processes and data and the creation of a "big picture."

Thus, comparisons were made only within the results of cultures of a single experimental batch and not among different experimental batches, despite the latter being the standard in conventional microbiology. To establish methodology, settings and formats compatible in markedly diverse applications in terms of fungal phenotypic phase, taxonomy, colony size, growth status, and environment were pursued whereas further optimizations are called for in the future with possible standardizations. As a result, non-standardized inocula and room temperature incubations were selected over precisely managed experimental settings, which constitute the default procedure in mycology experiments.

Fungi were selected over bacteria, because they are putative multicellular eukaryotes. The latter will allow for future iterations of comparative transcriptomics analysis more readily for basic research in electrogenomics/electrotranscriptomics (Siamoglou et al., 2020).

The selection of variables for initial testing was driven by concerns of applicability: Different substrates were tested to understand whether for experimentation in electroculturomics a substrate that tends to further growth would be preferable, and would lead to initial observation of differences and/or produce at a given time more visible differences in growth patterns. Different taxa were used, as the various applications envisaged depend on highly different fungal taxa, sometimes of a high order, as with Oomycota, whereas at other times of a very low order, as with species and subspecies levels (Kambouris and Velegraki, 2020). In addition, as fungi present unicellular and multicellular forms, they present a good system to identify mechanisms at cellular or higher level.

Different intensities were tested to detect optimal stimulus range. The direction of electrodes was added in the variables so as to explore the possibility of using complex treatment geometries to achieve directional growth or inhibition in complex environments, as in industrial equipment and urban habitats. Last, but not least, the frequency of treatment was tested. Single-treatment schemes would allow the processing of commercial goods at the inoculation phase, with ether priming or suppressing effects of the biochemical mechanism at the first stages of colony/mycelium formation in a new habitat. Daily treatment is the method of choice for selective targeted applications, both therapeutic and industrial. 


\section{Materials and Methods}

\section{Study design}

In this study, two different lines of experiments are presented: pilot experiments to discover areas for further experimentation and reject less promising approaches and factors; standard experiments in multiple so as to enable statistical analysis.

For the first line, a panel of fungi was used, as mentioned later, to plot time-effect (growth) curves after inoculation in two different substrates, either sabouraud dextrose agar (SDA) or malt extract agar (MEA) or both, so as to determine possible need for selection of one of them, the former being the standard choice for fungi in medical microbiology, the latter being richer in nutrients. The parameters tested were current intensity, treatment frequency, electrode positional geometry, and standard versus interchangeable polarity between successive treatment sessions. The exact experimental format is described later in the article, under the heading electrostimulation: device, conditions, procedure, and is summarized in Figure 1.

The second line of experiments included two fungi (Fusarium oxysporum and Colletotrichum gloeosporioides) in one substrate (SDA) treated in quadruple with multiple controls so as to allow statistical analysis. The factors were a narrow range of intensity settings ( 1 and $2 \mathrm{~mA}$ ) and treatment frequency (single treatment on inoculation and repeated, daily treatment) in a radial experimental design pattern (Fig. 2).

As the field is practically novel, a preparatory line of experiments was undertaken, to establish the optimal, in terms of effort and fidelity, untreated controls. The additional access to air was afforded by holes drilled in the dish, and the

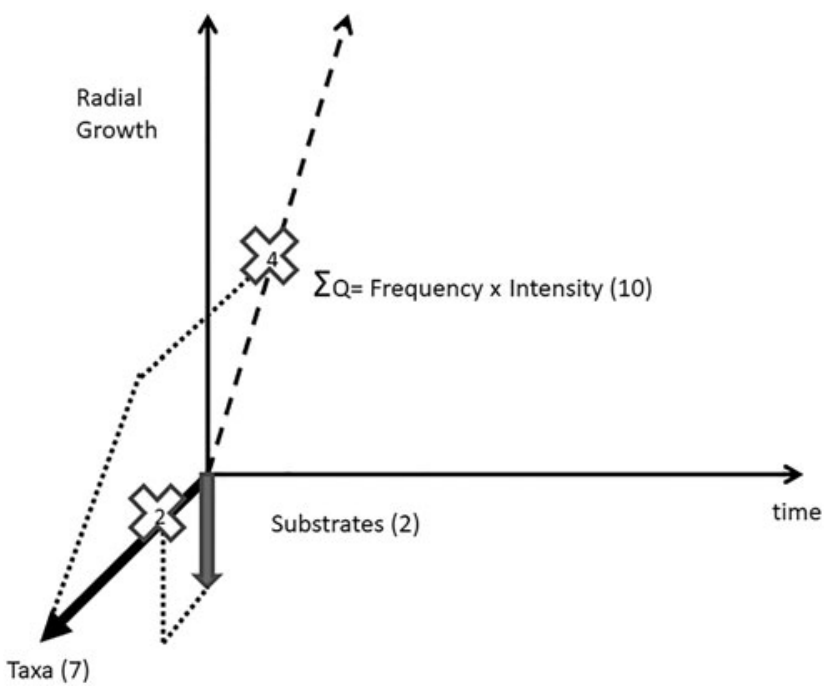

FIG. 1. The illustration of the key experiments. The development of growth (scored as the diameter in centimeters) over time (in days) was tested for a number of intensity settings (five different values) and frequencies of treatment (two different values) that can be summarized in one variable, accumulated charge $\left(\sum q\right)$. In four of these combinations, experiments were conducted with seven fungal taxa. Of these, two taxa were tested on a different substrate, MEA, instead of the SDA which was the standard for all other experiments. MEA, Malt Extract Agar; SDA, Sabouraud Dextrose Agar.

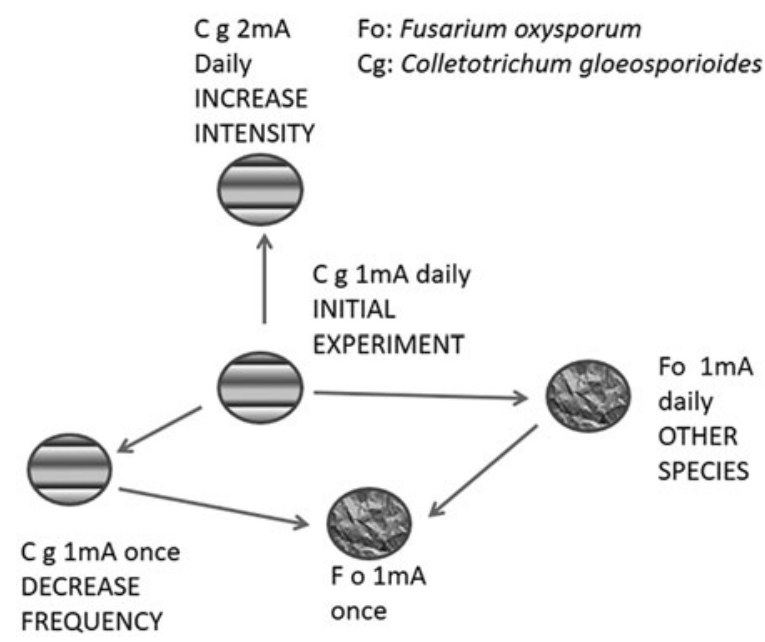

FIG. 2. The design of the experiments.

possible effect of the metal of the electrode in contact with the medium was examined.

All inoculation and treatment processes were carried out in a laminar flow cabinet and next to a Bunsen burner. This arrangement implies a double-layer safety setting since fungal airborne contaminants are ubiquitous. Holes drilled in the Petri dishes were sealed with parafilm after treatment and cleaned with $70 \%$ ethanol before and afterward, as with the electrodes.

\section{Experimental implementation}

Microorganisms and growth conditions. The fungi $F$. oxysporum and $C$. gloeosporioides were provided by The Division of Plant Protection of Patras (DPPP), identified by the Hellenic Collection of Pathogenic Fungi, University of Athens (UOA/HCPF), which provided all other fungal strains (Pythium ultimum, Rhizoctonia sp., Mucor sp., Rhizopus arrhizus, Candida parapsilosis, Cryptococcus neoformans, Aspergillus niger); all cultures were grown at room temperature in standard 9-cm Petri dishes with SDA with (UOA/HCPF) or without (DPPP) chloramphenicol (SDA-Chl), with two exceptions. One additional A. niger strain and the Mucor sp. were inoculated and grown on MEA standard 9-cm Petri dishes.

Petri dishes preparation for electrostimulation. After the addition of 20-mL medium (SDA or MEA) in each plastic Petri dish, solidification of medium, and marking of dishes, the latter were drilled for insertion of pin electrodes next to a Bunsen burner for asepsia.

Two spots, usually at $180^{\circ}$, but in some cases at $90^{\circ}$ and at $120^{\circ}$ were marked by a painter on the side of the dish. A microbiological steel needle of $0.2-\mathrm{cm}$ diameter was heated until red hot at the Bunsen burner $(\sim 40 \mathrm{sec})$ to meltpierce the plastic sidewall of the dish at one spot. After the first drilling, the needle was immersed in alcohol and heated again to drill the second hole of a dish, angled as mentioned earlier. During drilling, the needle must not puncture the medium in contact with the wall of the dish, and drilled dishes are kept as a stack near the Bunsen burner to preserve aseptic conditions. 
Inoculation process. Using sterile, disposable inoculating loops, a part of the yeast colony (or spores from mycelium) was taken from the initial culture and mixed with $800 \mu \mathrm{L}$ double-distilled water in an Eppendorf 1.8-mL microtube. The mix was agitated in vortex for $5 \mathrm{sec}$. By immersing another disposable inoculating loop, a drop was taken and inoculated in the center of Petri dish, which was subsequently closed and placed next to the Bunsen burner for $10 \mathrm{~min}$ for the drop to be absorbed before the treatment.

Determining the optimal control dish format. In a pilot experiment, three different pairs of controls were incubated after inoculation without treatment, to establish acceptable and/or proper forms of control for further use: One pair was intact, to establish a baseline; a second pair was drilled with two holes at $180^{\circ}$ to explore possible effects of the drilling (mainly in terms of aeration and drying); and a third pair was drilled and with electrodes inserted, but not attached to the power source to explore possible effects of the insertion of the electrodes and of the electrode material.

Electrostimulation: device, conditions, and procedure. The device used in this study was Mio Ionotens ${ }^{\circledR}$ from I-Tech Medical Division. This appliance generates transcutaneous electrical nerve stimulation (TENS) and ionophoresis currents and is provided with two independent and adjustable intensity channels. Provision and use of two connection cables with splitters (eight-electrode connection) afforded us the opportunity for four Petri dishes to be subjected to electrostimulation at the same time by pairs. Basic research experiments used for statistics were implemented by four identical dishes that were subjected to identical treatment in quantitative, qualitative, and temporal terms. Electrodes of each pair were hand-inserted to each plate before treatment onset (Fig. 3).
Each pilot experiment was conducted with the same growth medium and inoculum source and included five dishes: one untreated control dish and four treated ones, which was the limit of the potential of the device. The treated samples of a batch were divided into two pairs, each treated with a different intensity $(500 \mu \mathrm{A}, 1 \mathrm{~mA}, 2 \mathrm{~mA} 3.5 \mathrm{~mA}$, $5 \mathrm{~mA}$ ). Wave frequency was set at $1 \mathrm{~Hz}$, and wave impulse width was set at $250 \mu \mathrm{sec}$. One dish from each pair was treated once, on inoculation, and the other repeatedly, on a daily basis; treatment duration was always the same, either 10 or $15 \mathrm{~min}$. The dishes treated only once were left to incubate without further stimuli, but they were removed from the incubation emplacement for the time of the stimulation of the other pair, to emulate the non-electrodependent conditions.

For experiments on systematic treatment results, $F$. oxysporum and $C$. gloeosporioides were tested with a narrow range of parameters on SDA, in quadruple, which is the limit of the device. All four dishes were treated similarly, compared with the pairs of similar controls inoculated almost concurrently, and incubated identically.

The calculation of colony diameter was conducted for all dishes irrespective of treatment before the electrostimulation session of the day by marking the edges of the longer colony diameter with a fine marker to enhance accuracy and measuring the distance with a ruler. Before the beginning and after the end of electrostimulation, electrodes were cleaned by using $70^{\circ}$ ethanol. The Petri dishes were sealed by parafilm and incubated at room temperature off direct sunlight on completion of treatment sessions.

\section{Statistics}

Averages were scored separately for multiple treatments and the respective concomitantly incubated different kinds of controls and plotted on time-growth curves with standard

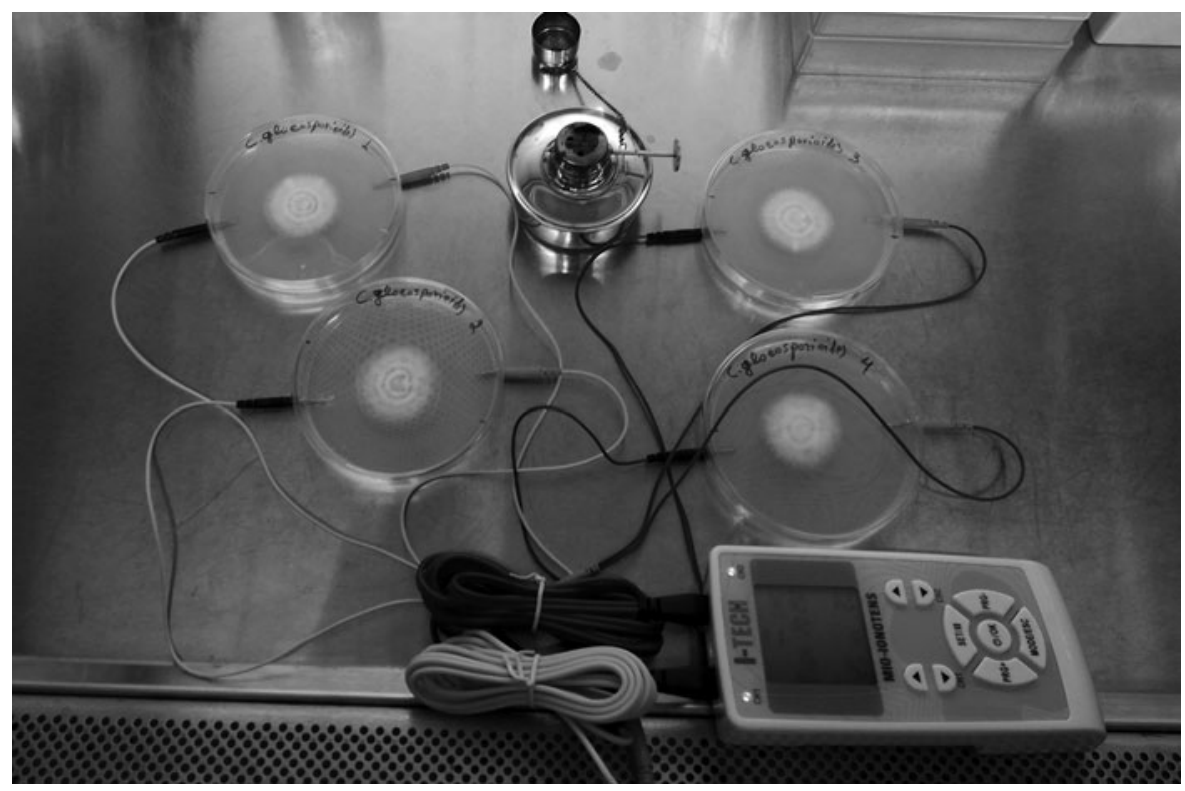

FIG. 3. The electrostimulation format in standard configuration, with one control and four simultaneous treatment dishes; the latter may be all identical or in two couples of two identical intensities. There is a Bunsen burner nearby, and the setting is in a laminar flow cabinet. 
deviation shown to represent inherent variability. Independent t-test was used in daily counting points to determine statistical significance of observed differences between control and treatment averages. $p$-Values of $<0.05$ were considered statistically significant.

\section{Results}

\section{The optimal format for untreated control dishes}

Petri dishes inoculated and incubated in ambient conditions in the standard way were compared with dishes punctured at the sides, similarly to treated dishes, to insert the electrodes. The two alternative settings, puncturedunpunctured, were tested in duplicate three times with $C$. gloeosporioides and two times with $F$. oxysporum (Supplementary Figs. S1 and S2) as part of the respective experiments. As is obvious from the standard deviation (SD) indicators, the growth of similar controls incubated in parallel is almost identical regardless of puncturing wholes.

In addition, SDA-Chl dishes with C. parapsilosis were observed in three conditions: (i) intact, (ii) punctured, and (iii) punctured with electrodes inserted but inert. The two last dishes of cases (ii) and (iii) had no difference whatsoever in growth diameter, whereas the intact dish showed a bit faster growth since day 4, which remained practically stable to the end of the experiment at day 7 (Supplementary Fig. S3).

\section{Systematic treatment outcome in fungal growth}

The determination of inherent variability in growth (as opposed to reproducibility) was tested by experimental sets of four (4) SDA dishes treated identically and simultaneously, against pairs of different controls (punctured/ unpunctured control dishes). Means computed for such fours and pairs were plotted to time-effect diagrams, the latter assessed as the maximum diameter of the mycelium/colony.

The inherent variability within the four identical dishes of an experimental run was shown to be minimal, as indicated by the standard deviation indicators in Figure 4A-E. The four identically treated dishes differed minimally. On the other hand, successive repeats, whenever tried, demonstrated limited reproducibility. The controls of $C$. gloeosporioides in experiments conducted on different days show different growth, as evident when comparing Figure 4A, B, and E.

C. gloeosporioides daily treatment at $1 \mathrm{~mA}$ showed less growth compared with the control; single-day treatment showed no difference compared with the control (Fig. 4A, B). The treatment of $F$. oxysporum, on the other hand, establishes a qualitative difference between daily and single treatment, and hence the necessity of both treatment methods. Daily treatment resulted in lower growth, whereas treatment once had a stimulatory effect (Fig. 4C, D). This stimulatory effect observed in only one of the two fungi exhaustively tested with very low accumulative dose proves dissimilar physiological indices among different Ascomycetes molds.

In terms of kinetics, the suppressive effect of treatment on C. gloeosporioides with $2 \mathrm{~mA}$ daily (Fig. 4E) started at day 1; treatment with $1 \mathrm{~mA}$ daily results in statistically significant differences in growth since day 8 (Fig. 4A), establishing a kinetic difference. The growth scores in control dishes used in the experiments with $1 \mathrm{~mA}$ treatments once and daily are similar; however, they were different from the controls of the
$2 \mathrm{~mA}$ daily treatment. This underscores the spontaneous variability of the fungus as an organism and the need for including proper, concomitant controls in every run.

A concept of cumulative dose would suggest that $2 \mathrm{~mA}$ daily is at the far end of a dose-effect peak, possibly implicating different mechanisms at the cellular level. One mechanism must have been activated early and be responsible for the stimulatory effect, possibly — and speculativelyassociated with membrane channels and exchange mechanisms. With additional charge accumulating, this same mechanism may be derailed. Alternatively, a different mechanism may be functioning over a certain accumulative threshold and incurring adverse effects. Such may be the conformational change of macromolecules; or their dislodging due to electrophoresis or dielectrophoresis (McGillivray and Gow, 1986; Siamoglou et al., 2020).

Mycelial qualitative phenotypes corroborate with what has been suggested earlier. In controls, there is better development of colored rings (Fig. 5A, B) and surface ripples (Fig. 5C, D) of C. gloeosporioides than in samples treated with $1 \mathrm{~mA}$ daily and just once, respectively.

The counts of the controls between the two experiments of F. oxysporum differ wildly (Fig. 4C, D). An inhibitive effect can be observed at day 8 in daily treated samples (Fig. 4C), whereas single-day treatment shows an inductive effect of a pervasive nature at day 5 , which is purely kinetic and lasts for 3 days, after which treatments and controls are on a par (Fig. 4D).

\section{Pilot experiments with multiple fungal taxa} and treatment intensities, polarities, and geometries

To detect possible lines of further application, a series of pilot experiments were conducted to simultaneously explore the differential effect of current intensity in a number of species, in different substrates, by using non-linear electrode geometry and while assessing the effect of repeated versus one-off treatment (treatment frequency). These experiments are summarized in Figure 1 and the respective results are depicted in Table 1.

\section{a. Electrode geometry}

The pilot treatment of C. parapsilosis dishes with $2 \mathrm{~mA}$ while the electrodes were inserted so as to form a $90^{\circ}$ or a $120^{\circ}$ angle with each other, instead of the usual $180^{\circ}$, has shown identical growth between treated dishes and the controls (Supplementary Fig. S4).

\section{b. Increased range of current intensity}

At $5 \mathrm{~mA}$, both C. parapsilosis and C. neoformans showed lower growth than the respective controls, whereas the substrate was partially liquidated making the counts unreliable (Fig. 6). The bubbles and foam have been solidified in different areas of the surface; thus, neither association with the electrodes nor electrolytic formation can be inferred. At $3.5 \mathrm{~mA}, C$. neoformans still showed lower growth than the control, whereas $C$. parapsilosis showed identical growth with its respective control. At $2 \mathrm{~mA}, C$. neoformans showed slightly lower growth than the respective control dish, but C. parapsilosis showed increased growth compared with the respective control dish (Supplementary Figs. S5 and S6). 

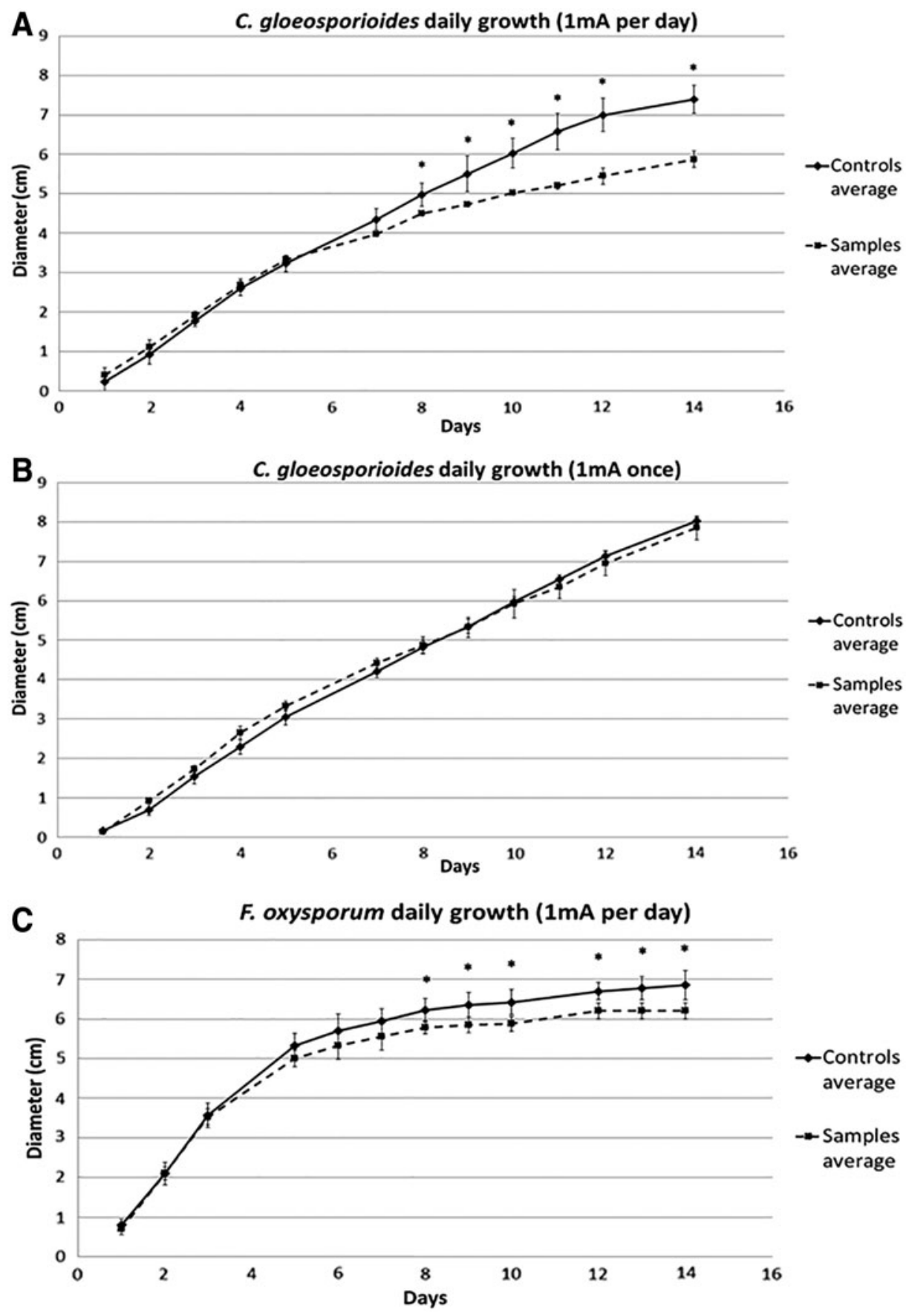

FIG. 4. (A) Colletotrichum gloeosporioides daily treatment at $1 \mathrm{~mA}$ showing a suppressive effect. $* p<0.05$, Controls versus Samples average. (B) C. gloeosporioides single-day treatment at $1 \mathrm{~mA}$ showing no appreciable effect. C. Fusarium oxysporum daily treatment at $1 \mathrm{~mA}$ showing a suppressive effect. ${ }^{*} p<0.05$, Controls versus Samples average. (D) $F$. oxysporum single-day treatment once at $1 \mathrm{~mA}$ showing a stimulatory effect. ${ }^{*} p<0.05$, Controls versus Samples average. (E) C. gloeosporioides daily treatment at $2 \mathrm{~mA}$ showing a suppressive effect. * $p<0.05$, Controls versus Samples average.

\section{c. Multiple fungal species tests}

The treatment of different fungal taxa with a limited range of treatment settings (two values of frequency: once and daily; and two of intensity: 0.5 and $1 \mathrm{~mA}$ ) is summarized in Table 1. $R$. arrhizus grown on SDA presented explosive growth, allowing one measurement, which, however, showed identical or nearly identical results between control and treated samples (Supplementary Fig. S7A).

Another member of the Zygomycota, a strain of Mucor sp., cultured on MEA showed more moderate growth and a very complicated pattern; daily treatment of $0.5 \mathrm{~mA}$ had beneficial effects and furthered growth, but $1 \mathrm{~mA}$ clearly not, suggesting a threshold somewhere in between either in terms of 

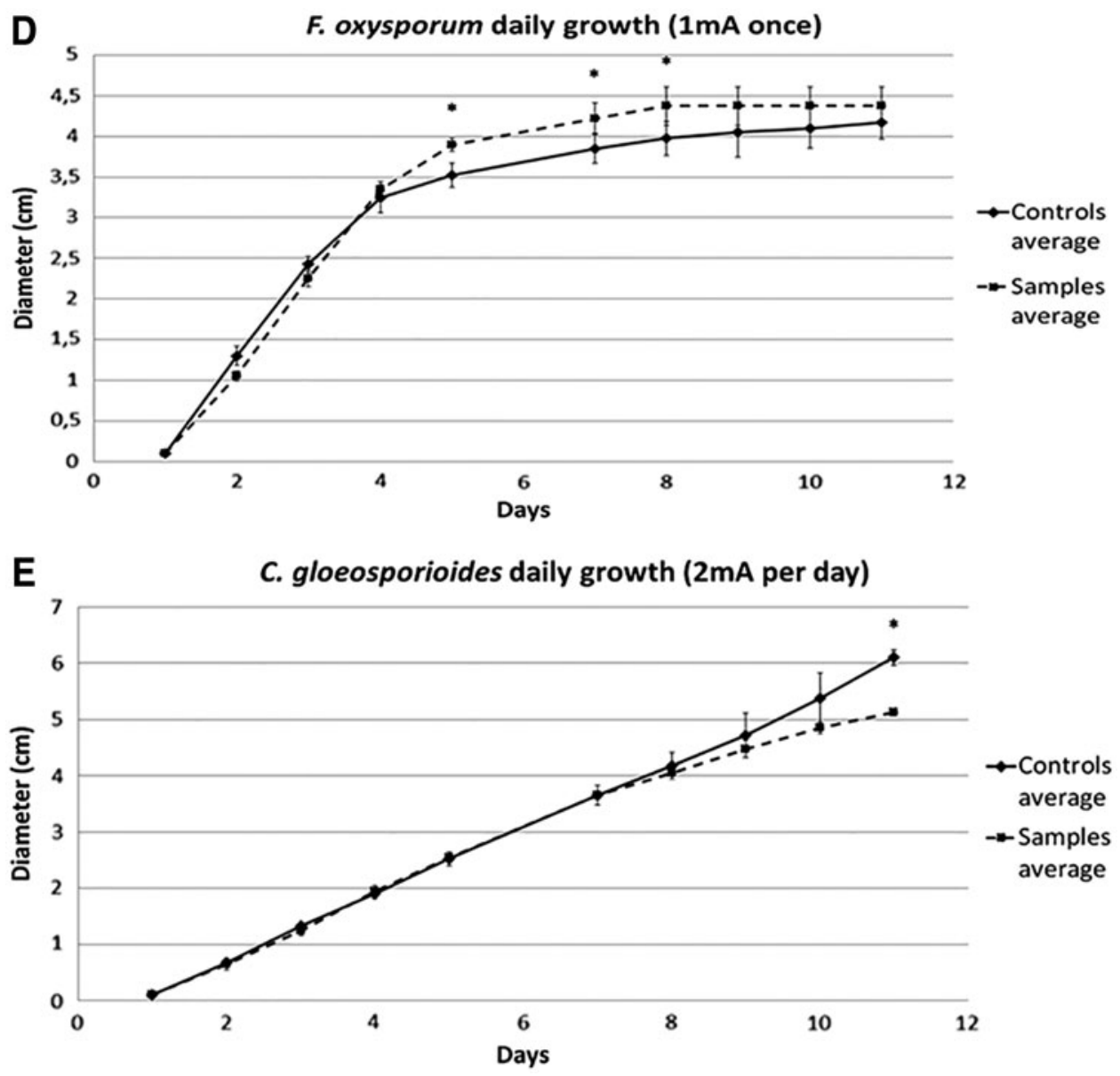

FIG. 4. (Continued).

intensity or in terms of accumulation (Supplementary Fig. S7B). But one-off treatments, which amounted to far less accumulated charges, showed drastically lower growth than the untreated control.

This contradiction may be explained by suggesting a deleterious initial effect that may be alleviated by a higher dose. The latter conceivably triggered a response/repair mechanism, which may account for the increased growth observed in daily treatment. Such complex interactions are speculative herein, but possible in terms of biological interpretation and underline the need for combined Omics studies, especially post-genomics, to elucidate the causality of the phenomena.

The Oomycota, $P$. ultimum, did show an increase in growth in all treatment conditions but for the application of $1 \mathrm{~mA}$ daily, where it eventually-but not initially-exhibited a decrease in growth (Supplementary Fig. S7C). A dosedependent effect is implied, with the respective treatment reaching a peak at the fifth dose of treatment, when the effect turned counterproductive if not outright restrictive.

The mycelial Basidiomycota (Rhizoctonia sp.) as shown in Supplementary Fig. S7D seems to possess a more complex mechanism, with kinetic rather than dynamic effects, which, however, differ by the intensity and frequency of treatment, as obvious at the 7th day of the experiment. C. neoformans (Basidiomycota yeast), on the other hand, showed much more subtle kinetic differentiations and a propensity for a decreased, not increased (as with the mycelial Basidiomycota), growth under treatment compared with the control (Supplementary Fig. S7E) and thus suggesting a $<0.5 \mathrm{~mA}$ optimum for conductive coupling.

The mycelial Ascomycota A. niger, under the same treatment produced very different results in two experiments, where different substrates (MEA and SDA-Chl) were used; still, the common denominator in both experiments has been the complex kinetics, which imply effects on the duration and onset of exponential growth.

In the two A. niger experiments, the growth rate of the control samples is highly different in the different substrates, as shown in Supplementary Figure S7F and G. This indicates the impact of the substrate at both spontaneous and stimulated growth rates, which comes as no surprise, as one of the two experiments was on SDA-Chl (Supplementary Fig. S7F) and the other on MEA (Supplementary Fig. S7G). The interesting issue is that the difference between spontaneous and stimulated growth is dissimilar as well. This means that the issue may be further followed by culturomics and resolved by integrated post-genomics, producing a true descriptive culturomics entity.

C. parapsilosis (Ascomycota yeast) at 0.5 and $1 \mathrm{~mA}$ has shown only slight growth increase under treatment and this with the lower intensity, insinuating in this case an intensity cutoff (Supplementary Fig. S7H). 

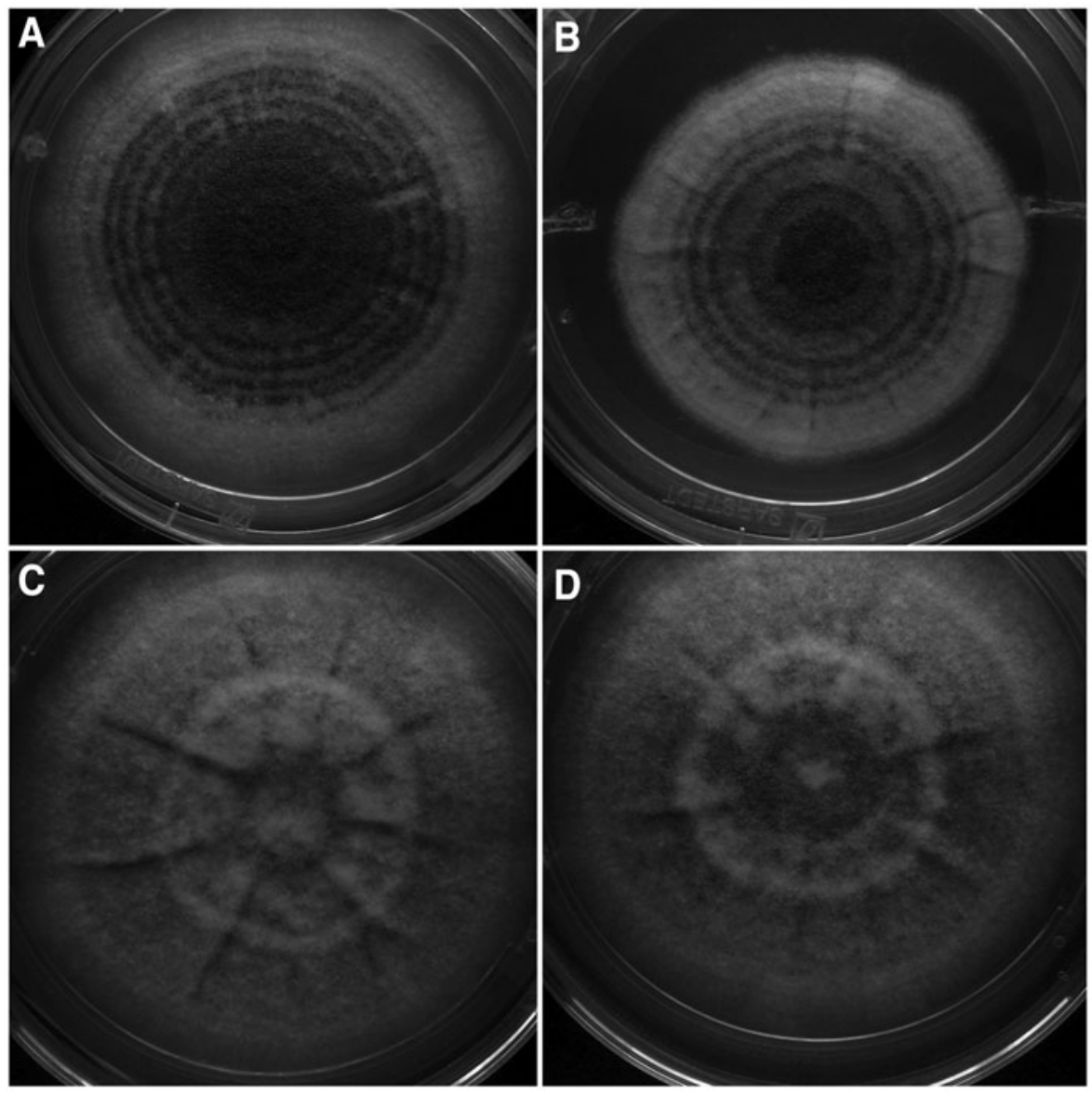

FIG. 5. Colored rings in Colletotrichum gloeosporioides control on day 13 (A) are better defined and sharper than those of the sample treated with $1 \mathrm{~mA}$ daily $(\mathbf{B})$. Surface radial ripples in C. gloeosporioides control on day $12(\mathbf{C})$ are deeper and better defined than those of the treated sample (D).

\section{Discussion}

We report here the introduction of a new research framework and technology platform for electroculturomics, by coupling standard solid-state mycological cultures with conductive treatment using a CE-certified medical ionophoresis device. We share our experience with a diverse range of fungi that have been treated with the electroculturomics approach reported herein. We suggest that this line of inquiry can be extended to electrotranscriptomics and electrometabolomics by deploying electroculturomics in tandem with multi-omics approaches in the future.

The most effective and precisely adjustable electrostimulation (ES) protocols are the conductive ones. Thus,

Table 1. Qualitative Synopsis of the Dose-Effect Results for Multiple Fungal Taxa in Two Substrates (Malt Extract Agar and Sabouraud Dextrose Agar)

\begin{tabular}{|c|c|c|c|c|c|c|c|c|}
\hline \multirow[b]{2}{*}{ Fungal taxa } & \multirow[b]{2}{*}{$S D A$} & \multirow[b]{2}{*}{$M E A$} & \multicolumn{2}{|c|}{$1 m A$} & \multicolumn{2}{|c|}{$0.5 \mathrm{Ma}$} & \multirow[b]{2}{*}{ Test dishes* } & \multirow[b]{2}{*}{ Control dishes } \\
\hline & & & Once & Daily & Once & Daily & & \\
\hline Mucor sp. & & $\mathrm{X}$ & - & - & - & + & 1 & 1 \\
\hline Rhizopus arrhizus & $\mathrm{X}$ & & 0 & 0 & 0 & 0 & 1 & 1 \\
\hline Pythium ultimum & $X$ & & $\mathrm{k}+$ & - & $\mathrm{k}+$ & $\mathrm{k}+$ & 1 & 1 \\
\hline Rhizoctonia sp. & $X$ & & $\mathrm{k}+$ & $\mathrm{k}+$ & $\mathrm{k}+$ & $\mathrm{k}+$ & 1 & 1 \\
\hline Aspergillus niger-1 & $\mathrm{X}$ & & $\mathrm{k}-$ & $\mathrm{k}-$ & $\mathrm{k}-$ & $\mathrm{k}+$ & 1 & 1 \\
\hline A. niger -2 & & $\mathrm{X}$ & - & + & - & - & 1 & 1 \\
\hline Cryptococcus neoformans & $X$ & & - & - & - & - & 1 & 1 \\
\hline Candida parapsilosis & $\mathrm{X}$ & & 0 & 0 & + & + & 1 & 1 \\
\hline
\end{tabular}

\footnotetext{
*Per treatment set.
}

-, suppressive effect; +, stimulatory effect; 0, no growth effect; k, kinetic nature of the observed effect; MEA, Malt Extract Agar; SDA, Sabouraud Dextrose Agar; X, the substrate used in the experiments. 


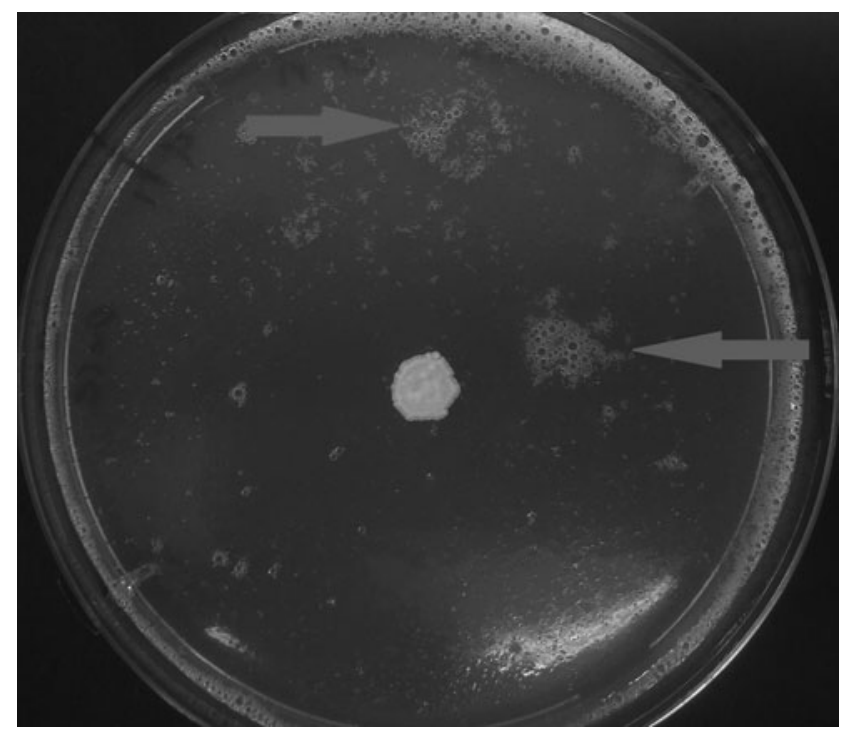

FIG. 6. Signs of liquidation (foam) on the surface of SDA dish (solid arrows) after $5 \mathrm{~mA}$ daily treatment of Cryptococcus neoformans on day 7.

their effect on microorganisms invited interest early on, and not only in a therapeutic capacity. Additional applications have been considered, such as:

- non-therapeutic sterilization, which is a vital issue in medicinal procedures and commodities such as catheters, lines, syringe needles scalpels, and bandages (Davis et al., 1991);

- bioengineering (Nakanishi et al., 1998; Sivasubramaniam and Franks, 2016);

- food and drug production/processing/conservation, exemplified by heifer and juice decontamination (Commission of the European Communities, 1987; Tracy, 1932);

- biotechnology applications; to avert microbial biodegradation or spoilage of useful stores and objects/ amenities and enhance decay and recycling of organic litter and sewage (Ranalli et al., 2002);

- biorestoration, and bioaugmentation of water and soil (Aracic et al., 2015; Olszanowski and Piechowiak, 2006).

Stimulatory effects of 0.1 to $0.3 \mathrm{~mA}$ direct currenet (DC) have been recorded on yeast growth since the early 20th century (Tracy, 1932). S. cerevisiae presented increased growth and ethanol production under $10 \mathrm{~mA} \mathrm{DC}$ (Nakanishi et al., 1998). Thus, it is a (not very) common secret that electrical modalities do not show stable and reproducible effects on microorganisms.

Such effects depend on many factors (growth phase/age, environmental conditions), including the special peculiarities and particularities of each strain, such as (indicatively but not exclusively) the spatiotemporal qualitative and quantitative distribution of polar molecules (McGillivray and Gow, 1986). Neither phylogenetic constants nor any continuous association/function have been established to date among relative taxa (Siamoglou et al., 2020) and the authors' educated guess is that post-genomic profiles, including but not restricted to metabolomics ones, may hold the key for such associations.
The different responses of $C$. neoformans and $C$. parapsilosis when treated with 3.5 and $2 \mathrm{~mA}$ are tell-tale and corroborated by a similar observation during the more meticulous testing of $C$. gloeosporioides and $F$. oxysporum exposed once at $1 \mathrm{~mA}$ (Fig. 4B, D), when the former showed no response whereas the latter was stimulated. Many of the observed growth differences are of a kinetic, not dynamic, nature, as is the brief stimulation of $F$. oxysporum when treated by $1 \mathrm{~mA}$ once (Fig. 4D). This makes the concepts of electrodynamics and electrokinetics more complex, compared with their originally intended use for merely characterizing the distribution, penetration, and effect of electrostimulation challenges onto patients in analogy to pharmacodynamics and pharmacokinetics (Kambouris et al., 2014, 2017).

Different treatment settings might produce more coherent and/or consistent results. The volume of tests combining settings and microorganisms, without even stating considerations of growth conditions (temperature, humidity, and substrate are just a few), is colossal effort. Obviously, very high-throughput methodology will be needed, supported by automation (Kambouris et al., 2018B) so as to agnostically mine associations, such as dose-effect curves in different conditions.

The in situ assembled multi-well plates with a conductive base are an existing piece of equipment that is compatible with advanced conductive formats, such as the non-contact current transfer (NCCT). Still, in this study an old-fashioned, rudimentary culturomics approach was selected with no custom-made equipment nor cutting-edge technology solutions, as traditional Petri dishes allow flexibility in design and implementation and enough space for enhanced discrimination in growth metrics. The choice of a certified, off-the-shelf medical instrument instead of a more presumptuous treatment solution, such as NCCT (Kambouris et al., 2017), allowed higher precision in tuning the current.

A chance finding was a high emergence of contamination in treated dishes compared with controls of the same batch (Table 2) and despite extensive aseptic provisions undertaken (laminar flow cabinet, Bunsen burner, aseptic techniques). Their distribution within the dishes precluded entry from the drilled holes, and their phenotypes precluded selfcontamination by veering spores during inoculation or during incubation. In addition, contaminations do not usually compromise growth tracking, as there are procedures to keep track of growth if an angular sector is contamination free.

Still, this prominence implies agents within the substrate that preferentially germinated and grew in treated dishes

Table 2. Comparative Incidence (+) or Absence (-) of Contamination by Fungus, Treatment INTENSITY, AND FREQUENCY

\begin{tabular}{lcccl}
\hline Fungus & Control & Once & Daily & Intensity \\
\hline Pythium ultimum & - & - & + & $1 \mathrm{~mA}$ \\
Cryptococcus & + & - & - & $5 \mathrm{~mA}$ \\
$\quad$ neoformans & & & & \\
C. neoformans & - & + & + & $2 ; 3.5 \mathrm{~mA}$ \\
Candida parapsilosis & - & + & + & $2 ; 3.5 \mathrm{~mA}$ \\
C. neoformans & + & + & + & $0.5 ; 1 \mathrm{~mA}$ \\
C. parapsilosis & - & + & - & $0.5 ; 1 \mathrm{~mA}$ \\
\hline
\end{tabular}


than in controls. Such a possibility should be taken into consideration whenever sterility is important. A healing mechanism induced by electric stimuli may conditionally nullify sterilization processes. It may well be similar to the mechanisms described for animal cells in the context of wound healing (Kambouris et al., 2014; Siamoglou et al., 2020).

The differential susceptibility of pathogens and host tissue and organs to various ES modalities might allow selective use against specific infections. Moreover, as there are many formats and a large number of electricity-based protocols already tried on different applications, including but not limited to ailments (Reid and Zhao, 2014), and a practically unlimited potential for developing new ones, there is an obvious issue of identifying and testing such differential applicability. Quite possibly, a range of applications is to be expected, wider than respective ranges for different biochemical compounds. This facilitates multi-tasking and furthers cost-effectiveness and sustainability while reducing costs and environmental footprints.

Roughly similar lines apply to all cases where suppression of fungi is required, as in disinfection, pasteurization, food conservation, building, and artefact maintenance. On the contrary, processes such as biodegradation, production of metabolites, and sewage treatment, which dictate enhanced fungal growth and perhaps tuning of the metabolism, similarly require exhaustive testing for enhancing only the desired species and strains in multi-species mycobiomes/ microbiomes (Kambouris and Velegraki, 2020).

This study was limited in scope, capabilities, and range. To follow daily treatments continuously, without mandatory gaps in treatment and counting due to weekends automation is advisable even for low-throughput formats.

A proper electroculturomics format would include additional parameters, each tested exhaustively, with additional indicative values and in a number of combinations and values tested simultaneously and at different multi-omics levels (genomic-post-genomic). Successive tests, even when identical in conditions and settings, may be misleading. Proper equipment, especially custom-designed consumables and plasticware, will allow increased precision in treatment. Results would ideally be collected automatically by scanning instrumentation with image processing software instead of the manual compilation of tables and spreadsheets and plotting of curves.

Electrotranscriptomics will elucidate the differential expression of genes under electrostimulation, interpreting the results of electroculturomics. Through electrogenomics, sequence variations will be assigned to different responses in treatment. Analogous macromolecules might react differently due to mutations, either directly (aminoacid sequence and cofactor variance) or indirectly (conformational variance due to aminoacid alterations). The basics have been determined earlier, when electrostimulation and electroregulation had been associated with different expression rates of specific genes (Gao et al., 2005; Meng et al., 2011; Zhao et al., 2006).

\section{Outlook and Conclusions}

Modern microbiology and drug development are in a watershed moment with the advent of electroceuticals. In addition to genomics, electrical impulses in an organism are believed to contribute to tissue and cellular plasticity. Hence, electroceuticals or bioelectronics offers the promise to identify innovative approaches to treat human diseases. However, applications toward electromicrobiology are still limited and rare, despite the high potential to innovate the fields of both microbiology and therapeutics. For example, electric modalities for manipulating microbial growth are highly sustainable, can be combined with biopharmaceuticals, probiotics, and pharmacobiotics, and thus are well poised for use in medicine, public health, ecology, and diverse industries.

We identified and described here several possible directions for future fundamental research in the nascent field of electroculturomics emerging from previous efforts in electromicrobiology, that is, the study of the effects of electric modalities on microbial life and growth. We identified specific and actionable research avenues showing the most promise for high-throughput formats. Indeed, the range of intensities used seemed to affect the fungal growth, at a minimum in terms of kinetics. Type, extent, and nature of the effects differed markedly, both by taxon and by substrate, although the latter needs further testing. Variability is very low among simultaneously performed and similarly treated cultures (Fig. 4A-E). This allows confidence in experimental terms.

Conversely, reproducibility is low among identical treatments on different days. The $C$. gloeosporioides controls showed different growth scores for similar day counts in successive applications (Fig. 4A, B, E), which poses challenges for practical applications and generalized protocols; further testing centered on relative effect rather than identical results is necessary.

In addition to the earlier observations, a proof of concept was achieved for conductive electrostimulation by pin electrodes inserted to standard Petri dishes. Thus, future hardware improving on the lines of the improvised setup used herein can be developed. In this respect, realizing that non-aligned (angled) electrodes showed no effect simplifies the design requirements. Similarly, identifying the proper form of control dishes for such experiments is also of importance for further, standardized, massive future use; since the three forms used show either no or negligible differentiation, any of the three can be used. Intact vessels are preferable for low cost or vessels with electrodes embedded but inert, for reasons of standardization in massive, multi-well formats may be selected according to the occasion.

Pilot experiments implied the association of intensity and frequency of treatment with differences in growth. This was an added module in this study, this time concerning basic research, with controls and treatments in multiple to allow statistical processing. The results support the idea that electrostimulation does affect growth in solid cultures, and that the effect may be inductive or suppressive for a given set of conditions, depending on the fungus. Age or even phase of growth of the inoculum may affect the outcome. It is plausible that the cell cycle phase, or growth phase, does affect the final result (Siamoglou et al., 2020).

Finally, there is a need for more extensive resources to identify the proper settings for growth manipulation on a case-by-case basis, thus introducing precision microbiology. 
Massively conducted experiments are essential for statistical robustness in the future.

This study is intended to make a specific contribution to fungal microbiology, and a broader contribution to advance the theory and practice of the field of electroculturomics in the context of 21st-century microbiology and ecology research.

\section{Author Disclosure Statement}

The authors declare they have no conflicting financial interests.

\section{Funding Information}

This work has been in part supported by the project "Synthetic Biology: From omics technologies to genomic engineering (OMIC-ENGINE)" (MIS: 5002636) which is implemented under "Reinforcement of the Research and Innovation Infrastructure," funded by the Operational Programme "Competitiveness, Entrepreneurship and Innovation" (NSRF 2014-2020) and co-financed by Greece and the European Union (European Regional Development Fund).

\section{Supplementary Material}

Supplementary Figure S1 Supplementary Figure S2 Supplementary Figure S3 Supplementary Figure S4 Supplementary Figure S5 Supplementary Figure S6 Supplementary Figure S7

\section{References}

Aracic S, Manna S, Petrovski S, Wiltshire JL, Mann G, and Franks AE. (2015). Innovative biological approaches for monitoring and improving water quality. Front Microbiol 6, 826.

Blaser MJ. (2014). The microbiome revolution. J Clin Invest 124, 4162-4165.

Commission of the European Communities. (1987). Investigation on the possible effect of electrical stimulation on $\mathrm{pH}$ and survival of foot-and-mouth disease virus in meat and offals from experimentally infected animals. Report EUR 10048 EN.

Davis CP, Wagle N, Anderson MD, et al. (1991). Bacterial and fungal killing by iontophoresis with long-lived electrodes. Antimicrob Agents Chemother 35, 21312134.

Famm K, Litt B, Tracey KJ, et al. (2013). Drug discovery: A jump-start for electroceuticals. Nature 496, 159.

Frinking E, Sweijs T, Sinning P, Bontje E, Della Frattina C, and Abdalla M. (2016). The Increasing Threat of Biological Weapons: Handle with Sufficient and Proportionate Care. Security 37, The Hague Center for Strategic Studies. The Hague.
Gao W, Liu Y, Zhou J, et al. (2005). Effects of a strong static magnetic field on bacterium Shewanella oneidensis: An assessment by using whole genome microarray. Bioelectromagnetics 26, 558-563.

Healthcare. (2008). Coverage position: Electrical stimulation for wound healing. https://my.cigna.com/teamsite/health/ provider/medical/procedural/coverage_positions/medical $/ \mathrm{mm}_{\text {_ }}$ 0351_coveragepositioncriteria_electrical_stimulation_for_ wound_healing.pdf. Accessed May 5, 2020.

Kambouris ME. (2020). Culturomics: The alternative from the past. In: Microbiomics: Dimensions, Applications, and Translational Implications of Human and Environmental Microbiome Research. Kambouris ME, and Velegraki A, eds. San Diego: Elsevier Inc./Academic Press, 155-174.

Kambouris ME, Gaitanis G, Manoussopoulos Y, et al. (2018c). Humanome versus microbiome: Games of dominance and pan-biosurveillance in the omics universe. OMICS 22, 528-538.

Kambouris ME, Kantzanou M, Arabatzis M, et al. (2018a). Rebooting bioresilience: A multi-OMICS approach to tackle global catastrophic biological risks (GCBRs) and next generation biothreats. OMICS 22, 35-51.

Kambouris ME, Markogiannakis A, Arabatzis M, et al. (2017). Wireless electrostimulation: A new approach in combating infection? Fut Microbiol 12, 255-265.

Kambouris ME, Pavlidis C, Skoufas E, et al. (2018b). Culturomics: A new kid on the block of phenomics and pharmacomicrobiomics for personalized medicine. OMICS 22, 108-118.

Kambouris ME, and Velegraki A. (2020). Myc(et)obiome: The big uncle in the family. In: Microbiomics: Dimensions, Applications, and Translational Implications of Human and Environmental Microbiome Research. Kambouris ME, and Velegraki A, eds. San Diego: Elsevier Inc./Academic Press, 29-52.

Kambouris ME, Zagoriti Z, Lagoumintzis G, et al. (2014). From therapeutic electrotherapy to electroceuticals: Formats, applications and prospects of electrostimulation. Ann Res Rev Biol 4, 3054-3070.

Kloth LC. (2005). Electrical stimulation for wound healing: A review of evidence from in vitro studies, animal experiments, and clinical trials. Int $\mathbf{J}$ Low Extrem Wounds 4, 23-44.

Koblentz GD. (2017). The de novo synthesis of horsepox virus: Implications for biosecurity and recommendations for preventing the re-emergence of smallpox. Health Security 15, 620-628.

McGillivray AM, and Gow NA. (1986). Applied electrical fields polarize the growth of mycelial fungi. J Gen Microbiol 132, 2515-2525.

Meng S, Rouabhia M, and Zhang Z. (2011). Electrical stimulation in tissue regeneration. In: Applied Biomedical Engineering. Gargiulo G, and McEwan A, eds. London: IntechOpen, 38-63.

Nakanishi K, Tokuda H, Soga T, et al. (1998). Effect of electric current on growth and alcohol production by yeast cells. J Ferment Bioeng 85, 250-253.

Olszanowski A, and Piechowiak K. (2006). The use of an electric field to enhance bacterial movement and hydrocarbon biodegradation in soils. Polish J Environ Stud 15, 303-309. 
Pikaar I, Matassa OS, Rabaey K, et al. (2017). Microbes and the next nitrogen revolution. Environ Sci Technol 51, 7297 7303.

Poltawski L, and Watson T. (2009). Bioelectricity and microcurrent therapy for tissue healing-A narrative review. Phys Ther Rev 14, 104-114.

Ramadhinara A, and Poulas K. (2013). Use of wireless microcurrent stimulation for the treatment of diabetesrelated wounds: 2 Case reports. Adv Skin Wound Care 26, $1-4$.

Ranalli G, Iorizzo M, Lustrato G, et al. (2002). Effects of low electric treatment on yeast microflora. J Appl Microbiol 93, 877-883.

Reid B, and Zhao M. (2014). The electrical response to injury: Molecular mechanisms and wound healing. Adv Wound Care (New Rochelle) 3, 184-201.

Rowley BA, McKenna JM, and Wolcott LE. (1974). Proceedings: The Use of Low Level Electrical Current for Enhancement of Tissue Healing. Biomed Sci Instrum 10, 111-114.

Siamoglou S, Boltsis I, Chassomeris CA, et al. (2020). Electromagnetism and the microbiome(s). In: Microbiomics: Dimensions, Applications, and Translational Implications of Human and Environmental Microbiome Research. Kambouris ME, and Velegraki A, eds. San Diego: Elsevier Inc./Academic Press, 299-332.

Sivasubramaniam D, and Franks AE. (2016). Bioengineering microbial communities: Their potential to help, hinder and disgust. Bioengineered 7, 137-144.

Tracy RL. 1932. Lethal effect of alternating current on yeast cells. J Bacteriol 24, 423-438.

Whipps JM, Lewis K, Cooke RC. (1988). Mycoparasitism and plant disease control. In: Fungi in Biological Control Systems. Burge MN, ed. Manchester: Manchester University Press, 161-187.
Wiles S. (2015). All models are wrong, but some are useful: Averting the 'microbial apocalypse. Virulence 6, 730-732.

Zhao M, Song B, Pu J, et al. (2006). Electrical signals control wound healing through phosphatidylinositol-3-OH kinasegamma and PTEN. Nature 442, 457-460.

Address correspondence to: Manousos E. Kambouris, PhD Laboratory of Pharmacogenomics and Individualized Therapy

Department of Pharmacy School of Health Sciences University of Patras Patras 26500 Greece

E-mail: mekambouris@yahoo.com

$\begin{aligned} & \text { Abbreviations Used } \\ & \mathrm{CE}=\text { conformite Européene } \\ & \mathrm{DC}=\text { direct control } \\ & \mathrm{DPPP}=\text { Division of Plant Protection of Patras } \\ & \mathrm{ES}=\text { electrostimulation } \\ & \mathrm{MEA}=\text { malt extract agar } \\ & \mathrm{NCCT}=\text { non-contact current transfer } \\ & \mathrm{SD}=\text { standard deviation } \\ & \mathrm{SDA}=\text { sabouraud dextrose agar } \\ & \text { SDA-Chl }=\text { SDA chloramphenicol } \\ & \text { TENS }=\text { transcutaneous electrical nerve stimulation } \\ & \text { UOA/HCPF }=\text { Hellenic Collection of Pathogenic Fungi, } \\ & \text { University of Athens }\end{aligned}$

